\title{
Continuous rocuronium administration for tracheomalacia
}

\author{
Takeshi Yano $^{1} \cdot$ Masahiko Taniguchi $^{1} \cdot$ Isao Tsuneyoshi $^{1}$
}

Received: 5 March 2015 / Accepted: 13 July 2015 / Published online: 30 July 2015

(C) Japanese Society of Anesthesiologists 2015

\section{To the Editor:}

We describe two cases associated with incidences of life-threatening hypoxemia due to tracheomalacia that were repressed by rocuronium. Case 1: A 6-month-old female was admitted to hospital due to cyanosis. Most of the severe hypoxic episodes were life threatening and required cardiac massage during mechanical ventilation. Exacerbation of the airway obstruction with contraction of the thoracic and abdominal muscles was observed during computed tomography imaging (see Fig. S1). We decided on continuous intravenous administration of $5-8 \mu \mathrm{g} / \mathrm{kg} / \mathrm{min}$ of rocuronium to prevent further cyanotic episodes. After rocuronium administration, no additional life-threatening events occurred. Case 2: A 4-month-old female with a history of severe hypoxic episodes due to tracheomalacia was admitted to hospital. In accordance with our experience in case 1, we decided to administer continuous intravenous administration of rocuronium. No subsequent airway complications occurred after rocuronium was started. In both cases, rocuronium were reduced after tracheostomy. Mechanical ventilation was continued and planned to be maintained even after discharge from the hospital.

Electronic supplementary material The online version of this article (doi:10.1007/s00540-015-2055-3) contains supplementary material, which is available to authorized users.

\footnotetext{
Takeshi Yano

yanotake@med.miyazaki-u.ac.jp

1 Department of Anesthesiology and Intensive Care, Faculty of Medicine, University of Miyazaki, 5200 Kihara, Kiyotake, Miyazaki 889-1692, Japan
}

Symptoms may be exacerbated by activities that increase a child's respiratory efforts, including coughing and crying, which can increase intrathoracic pressure until the extraluminal pressure exceeds the intraluminal pressure, and the trachea collapses [1]. Hypoxic episodes might be prevented by reducing the increase of intrathoracic pressure due to intercostal and abdominal muscle contraction during coughing and crying, as in our cases. In conclusion, we recommend using rocuronium for ventilated patients as a pharmacological support for the prevention of life-threatening hypoxic episodes due to severe tracheomalacia.

\section{Compliance with ethical standards}

Conflict of interest None.

\section{Reference}

1. Carden KA, Boiselle PM, Waltz DA, Ernst A. Tracheomalacia and tracheobronchomalacia in children and adults: an in-depth review. Chest. 2005;127:984-1005. 